\title{
The Time Course of Name Retrieval During Multiple-Object Naming: Evidence From Extrafoveal-on-Foveal Effects
}

\author{
Debra Malpass \\ University of Birmingham
}

\author{
Antje S. Meyer \\ University of Birmingham and \\ Max Planck Institute for Psycholinguistics
}

\begin{abstract}
The goal of the study was to examine whether speakers naming pairs of objects would retrieve the names of the objects in parallel or in sequence. To this end, we recorded the speakers' eye movements and determined whether the difficulty of retrieving the name of the 2 nd object affected the duration of the gazes to the 1st object. Two experiments, which differed in the spatial arrangement of the objects, showed that the speakers looked longer at the 1st object when the name of the 2nd object was easy than when it was more difficult to retrieve. Thus, the easy 2 nd-object names interfered more with the processing of the 1st object than the more difficult 2nd-object names. In the 3rd experiment, the processing of the 1st object was rendered more difficult by presenting it upside down. No effect of 2nd-object difficulty on the gaze duration for the 1st object was found. These results suggest that speakers can retrieve the names of a foveated and an extrafoveal object in parallel, provided that the processing of the foveated object is not too demanding.
\end{abstract}

Keywords: speech production, visual attention, object naming, eye movements

Several studies have shown that common objects easily activate their names, even when the viewer has no intention of naming them. For instance, Morsella and Miozzo (2002) presented their participants with target pictures, shown in green, and superimposed distractor pictures, shown in red. The participants were faster to name targets accompanied by distractors with phonologically similar names (e.g., target: bed; distractor: bell) than targets accompanied by unrelated distractors (e.g., bed-hat). Similar findings were obtained by Kuipers and La Heij (2009), Meyer and Damian (2007), Navarrete and Costa (2005), and Roelofs (2008b; but see Jescheniak et al., 2009). However, when people speak, they normally produce only the intended words in the intended order. Therefore, there must be control mechanisms that increase the activation levels of relevant linguistic units relative to those of irrelevant units.

Different proposals concerning the nature of these control mechanisms have been made. For instance, theories of sentence generation often assume that speakers generate structural frames specifying in which order words of different syntactic categories must appear in an utterance. Words meeting the syntactic criteria receive additional activation in the correct order (e.g., Dell, 1986; Dell, Burger, \& Svec, 1997; Dell, Oppenheimer, \& Kittredge, 2008; Janssen, Alario, \& Caramazza, 2008; MacKay, 1987). The present

Debra Malpass, School of Psychology, University of Birmingham, Birmingham, England; Antje S. Meyer, School of Psychology, University of Birmingham, and Max Planck Institute for Psycholinguistics, Nijmegen, the Netherlands.

This research was supported by Economic and Social Research Council Grant RES-000-23-1508 to Antje S. Meyer.

Correspondence concerning this article should be addressed to Antje S. Meyer, Max Planck Institute for Psycholinguistics, P.O. Box 305, Nijmegen, NL 6500 AH, the Netherlands. E-mail: antje.meyer@mpi.nl study concerns the role of visual attention in controlling the activation levels of the visual-conceptual representations of objects and of the associated names. Directing visual attention at a single object at a time or distributing visual attention across several objects may be ways of controlling the activation levels of the object names. Adopting a narrow focus of attention should minimize interference between object names, whereas using a wider focus (processing several objects in parallel) might enhance speech fluency. In the experiments described below, we asked speakers to name pairs of objects in a fixed order using bare nouns, such as "duck, ladder." To study their allocation of visual attention, we recorded their eye movements while they prepared and produced the utterances. Our main questions were when and for how long the speakers would look at and attend to each object, and whether the names of the objects would become activated in parallel or sequentially. Before describing the experiments, we review earlier eyetracking studies that motivated the present research.

\section{Allocation of Visual Attention in Multiple-Object Naming}

Many studies have used eye tracking to investigate how speakers coordinate visual information processing, speech planning, and articulation while describing events, scenes, or sets of objects. In a seminal study, Griffin and Bock (2000) investigated the gaze patterns of speakers describing cartoons of events in utterances such as "The mailman is chasing the dog" (see also BrownSchmidt \& Konopka, 2008; Brown-Schmidt \& Tanenhaus, 2006; Gleitman, January, Nappa, \& Trueswell, 2007; Papafragou, Hulbert, \& Trueswell, 2008). In other studies, which are more relevant to the present research, speakers named small sets of objects in a prespecified order (e.g., from left to right). These studies revealed that the speakers typically fixated on each object before mentioning it and that their eye movements were tightly coordinated with 
their speech planning. The processes involved in naming an object can be parsed into visual-conceptual processes leading to the identification of the object, the selection of a lexical unit (called a lemma here), word-form encoding, and, finally, articulation. Word-form encoding occurs in several steps, namely morphological, phonological, and phonetic encoding (e.g., Levelt, 1989; Levelt, Roelofs, \& Meyer, 1999; see also Caramazza, 1997; Johnson, Paivio, \& Clark, 1996). The eye-tracking studies have shown that the time speakers spend looking at each object (the gaze duration) depends not only on the time they need to identify the object (Griffin \& Oppenheimer, 2006; Meyer, Sleiderink, \& Levelt, 1998) but also on the time required to retrieve the lemma and generate the phonological form of the name (Belke \& Meyer, 2007; Griffin, 2001; Meyer \& van der Meulen, 2000). For instance, several experiments have demonstrated that speakers look longer at objects with long names than at objects with shorter names (e.g., Korvorst, Roelofs, \& Levelt, 2006; Meyer, Belke, Häcker, \& Mortensen, 2007; Meyer, Roelofs, \& Levelt, 2003; but see Griffin, 2003). This indicates that speakers only initiate the shift of gaze to a new object after they have begun to retrieve the word form for the present object (Roelofs, 2007, 2008a).

There is strong evidence for a tight coupling between eye movements and visual attention, with each saccade being preceded by a corresponding shift of the focus of visual attention (e.g., Deubel \& Schneider, 1996; Hoffman \& Subramaniam, 1995; Irwin, 2004; Irwin \& Gordon, 1998). The shift of visual attention occurs around the time that the programming of the saccade begins, and it involves related neural circuits (e.g., Awh, Armstrong, \& Moore, 2006; Eimer, van Velzen, Gherri, \& Press, 2007; Ikkai \& Curtis, 2008; McDonald, 2006). Assuming that the time needed to program a saccade is fairly constant, the results of the eye-tracking studies reviewed above imply that speakers begin to plan a saccade and direct their attention to a new object when the phonological encoding of the name of the present object begins (Roelofs, 2007, 2008a). In other words, the speaker's visual attention is focused on the present object until its name has been planned to the level of the phonological form and only then moves on to the next object. A likely reason for the late shift of visual attention is that attending to an object facilitates not only its identification but, through a process of spreading activation, also the retrieval of the associated lexical information (e.g., Humphreys \& Forde, 2001; Humphreys, Riddoch, \& Price, 1997; Roelofs, 1992; see also Griffin, 2004; Wühr \& Frings, 2008; Wühr \& Waszak, 2003). Thus, speakers planning to name two objects in a specific order implement this plan by directing their visual attention initially to the first and then to the second object. Consequently, the conceptual representations of the objects and the associated lexical representations receive additional activation in the same order, which facilitates their selection and the generation of the correct word order.

However, a person's point of gaze does not reveal whether their attention is focused on a single object or whether he or she attends to several objects in parallel. The results of the eye-tracking studies mentioned above do not reveal whether speakers first exclusively attended to the first and then exclusively to the second object, or whether they initially directed the focus of their visual attention to the first object but simultaneously allocated some attention to the second object and processed it with a lower priority (e.g., Cave \& Bichot, 1999; LaBerge \& Brown, 1989; for discussions of serial vs. parallel processing of words during reading and reviews of the relevant evidence, see Engbert, Nuthmann, Richter, \& Kliegl, 2005; Kennedy, 2000; Rayner, 1998; Reichle, Rayner, \& Pollatsek, 2003). Two earlier studies_-by Morgan and Meyer (2005) and by Meyer, Ouellet, and Häcker (2008)—aimed to distinguish between these options.

In Morgan and Meyer's (2005) study, participants named triplets of objects in the order left-right-bottom object. During the saccade from the left to the right object, the right object seen at trial onset (the interloper) was replaced by a new object. This was the target that the participants had to name. The relationship between interloper and target was varied: They were unrelated objects, identical objects, or conceptually unrelated objects with homophonous names (e.g., animal/baseball bat). Morgan and Meyer found that gazes to the target were shorter when interloper and target were identical or had homophonous names than when they were unrelated (see also Pollatsek, Rayner, \& Collins, 1984).

One account of these findings is that the speakers processed the interloper in parallel with the foveated object and that the processing of the target was facilitated when it was identical to the interloper or shared the name. However, the results are open to an alternative account. Because eye movements are preceded by corresponding shifts of visual attention, it is possible that the speakers first attended exclusively to the left object and thenshortly before the onset of the eye movement-moved the focus of their visual attention and exclusively attended to the right object. Therefore, the interloper effects could arise while the speakers were still looking at the left object, but their visual attention was already focused on the right object.

However, results obtained by Meyer et al. (2008) argue against the latter view. Meyer et al. used the same experimental paradigm as Morgan and Meyer (2005) and very similar interloper-target pairs. Additionally, the difficulty of the left object, which was to be mentioned first, was varied. As in Morgan and Meyer's study, the gaze durations for the targets (the right objects) were shorter when the targets were preceded by identical or homophonous interlopers than by unrelated interlopers. However, these preview effects were significantly smaller when the left object was difficult than when it was easier to process. This interaction supports the view that the two objects were processed in parallel and competed for processing resources: When the left object was difficult to process, fewer resources were available to process the right object than when the left object was easier to process. This led to smaller preview benefits in the difficult than in the easy left-object condition. If the speakers first attended exclusively to the left object and then exclusively to the right object, the difficulty of the left object should not have affected the processing of the interloper and moderate its effect on the processing of the target.

This argument is based on the assumption that the timing of the saccade from the left to the right object relative to the shift of visual attention was the same for easy and difficult left objects. This is plausible, given the evidence mentioned above that shifts of visual attention are linked to saccade programming. We know of no evidence suggesting that the time required to program a saccade depends on the difficulty of processing the foveated stimulus. Nevertheless, one might speculate that the shift of visual attention to the right object occurred earlier during the processing of an easy left object (e.g., after the object had been recognized) than during the processing of a more difficult left object (e.g., after its name 
had been selected), whereas the saccade was always initiated at the same time (e.g., after the phonological form of the object name had been retrieved). The time interval during which the focus of attention was on the right object but the left object was still fixated on would then be longer for easy than for difficult left objects. This could account for the difference in the size of the preview effects (for a similar argument concerning preview effects in reading, see Henderson \& Ferreira, 1990; Reichle, Pollatsek, Fisher, \& Rayner, 1998). In short, it cannot be determined whether speakers attended to the two objects in parallel or whether the shift of covert visual attention to the right object occurred earlier when the left object was easy than when it was more difficult to process.

\section{The Present Study}

In the present study, we used a different paradigm to assess the allocation of visual attention and the time course of name retrieval in the multiple-object naming task. Adopting a research strategy from studies of reading (e.g., Inhoff, Starr, \& Shindler, 2000; Kennedy, 2000), we examined whether properties of an extrafoveal object affected the time that speakers needed to process a foveated object. On each trial of Experiment 1, two objects were presented next to each other, and the participants were asked to name first the left and then the right object. The right object was either easy or more difficult to name. We examined whether this affected the duration of the gazes to the left object. If speakers process the right object before they initiate the shift of gaze toward it, and if the processing is sufficient to activate the name of the right object, competition might arise between the names of the left and right objects, such that the left object would be processed more slowly when the right object was difficult than when it was easier to process. Alternatively, an easy right object should activate the associated conceptual and linguistic representations more rapidly than a more difficult one and might therefore interfere more with the processing of the left object. Regardless of its direction, an extrafoveal-on-foveal effect-that is, an effect of the difficulty of the extrafoveal right object on the duration of the gazes to the left object-must arise before the saccade to the right object is initiated. It would either indicate that the two objects were processed in parallel or that the shift of visual attention from the left to the right object occurred early enough for the lexical access processes for the two object name to overlap in time.

The results obtained by Meyer et al. (2008) show that the extrafoveal objects were processed more efficiently when the foveated objects were easy than when they were more difficult to process. To maximize the likelihood of obtaining an extrafovealon-foveal effect, we designed the present study such that the left object (which was fixated at trial onset) would be very easy to recognize and name. Sixteen objects were selected for the left position, but in each test block, only four objects were shown, five times each. The repeated presentation of a small set of items should render the objects easy to identify and name (e.g., Francis, Corral, Jones, \& Sáenz, 2008).

There were two types of blocks: homogeneous blocks, in which the four left objects were members of the same semantic category, and heterogeneous blocks, in which they were from different categories. Several studies have shown that speakers are slower to name an object (e.g., a chair) after having named other objects from the same semantic category (e.g., table, wardrobe, bed) than after having named unrelated objects (e.g., Damian, Vigliocco, \& Levelt, 2001; Howard, Nickels, Coltheart, \& Cole-Virtue, 2006; Kroll \& Stewart, 1994). This semantic blocking effect is maintained even when unrelated objects are named between objects from the same category (e.g., Damian \& Als, 2005; Howard et al. 2006). Most likely, the semantic blocking effect arises during lemma retrieval - that is, when speakers select suitable names for the objects from the mental lexicon (e.g., Belke, 2008; Damian et al., 2001; Howard et al., 2006; Kroll \& Stewart, 1994; Vigliocco, Vinson, Damian, \& Levelt, 2002). We expected the speakers to be slower to name the left objects and look at them for a longer time in homogeneous than in heterogeneous blocks.

There were two reasons for using homogeneous and heterogeneous blocks. First, if no effect of right-object difficulty on the gaze durations for the left objects was obtained, it would be useful to demonstrate that the left-object gaze durations were systematically affected by another variable. This would rule out that the absence of an extrafoveal-on-foveal effect was due to technical problems. Second, we could assess whether the variation in the difficulty of selecting the names of the left objects interacted with the effect of right-object difficulty. On the basis of the findings reported by Meyer et al. (2008), one would predict the participants to process the right objects more efficiently when access to the names of the left objects is easy (in the heterogeneous condition) than when it is more difficult (in the homogeneous condition). The extrafoveal-on-foveal effect should therefore be stronger in the homogeneous condition than in the heterogeneous condition.

For the right position, we selected 24 easy and 24 difficult objects. Pretests that used object recognition and naming tasks had shown that the two sets of objects differed in the ease of name retrieval but not in the ease of object recognition (see below for details). An extrafoveal-on-foveal effect found with these materials would indicate that the participants not only recognized the right object but also retrieved its name before initiating the saccade toward it.

Although the right objects had been pretested, it was important to establish that the participants of the main experiment would find the easy right objects easier to process than the difficult ones. Therefore, the gaze durations for the right objects were measured. However, the gaze duration for an object is a meaningful indicator of processing difficulty only if the viewer has to fixate on another stimulus as soon as the processing of the object has been completed. Therefore, an arrow was presented at the bottom of the screen (see Figure 1), and participants were asked to name the objects and then to indicate by pressing one of two buttons whether the arrow pointed to the left or to the right. Because the arrow was small and flanked by number symbols, the participants had to fixate on it to identify it. Several earlier studies (Meyer et al., 2003; Roelofs, 2007, 2008a) that used the same combination of tasks-object naming followed by symbol categorization-showed that participants only initiated the eye movement to the symbol after they had retrieved the phonological form of the object name. Thus, the gaze duration for the right objects is a good measure of the time required to identify the objects and to retrieve their names.

In Experiment 1, the two objects were shown next to each other. In Experiment 2, the second object appeared below the first object, and the arrow appeared on the right side of the screen. Finally, in 
Experiment 1

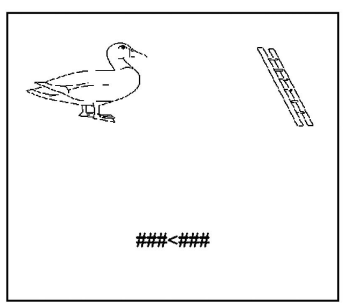

Experiment 2

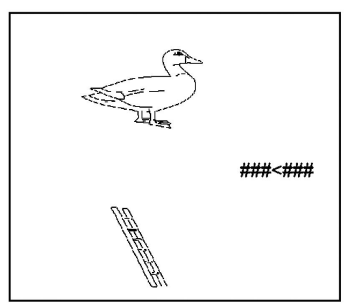

Experiment 3

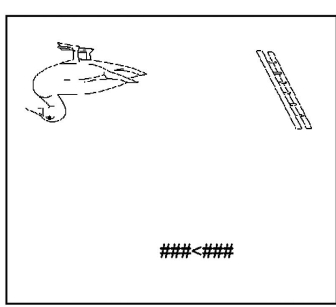

Figure 1. Arrangement of objects in Experiments 1-3.

Experiment 3, the left object was shown upside down. The reasons for conducting these experiments are given below.

\section{Experiment 1}

\section{Method}

Participants. The experiments were conducted with students of the University of Birmingham, who were paid or received course credits for their participation. They were native speakers of British English and had normal or corrected-to-normal vision. Experiment 1 was carried out with 24 participants.

Materials. On each trial the participants named two line drawings of objects shown next to each other. For the left position, 16 objects, stemming from four semantic categories (animals, furniture, tools, and vehicles), were selected. All left objects had monosyllabic names with an average frequency of 36.75 occurrences per million (University of Birmingham's, 2000, COBUILD Corpus of English Sentences database; see the Appendix for a listing of the materials).

For the right position, we selected two sets of 24 objects each, called the easy set and difficult set hereafter, which were matched for ease of object recognition but differed in the ease of name retrieval. Two pretests were carried out to select these objects. In the first pretest, 24 participants named line drawings of $100 \mathrm{ob}-$ jects. The easy and difficult objects were selected on the basis of the average naming latencies and error rates. The mean pretest naming latencies were $666 \mathrm{~ms}(S D=76 ; 1.08 \%$ errors $)$ and 818 $\mathrm{ms}(S D=93 ; 3.85 \%$ errors $)$ for the easy and difficult sets, respectively, $t_{1}(23)=15.04, p<.001 ; t_{2}(46)=9.80, p<.001$. The names of the easy objects had a frequency of 150.89 occurrences per million $(S D=125.46)$ and an average length of 1.25 syllables $(S D=0.44)$. The names of the difficult objects had a frequency of 26.37 occurrences per million $(S D=39.74)$ and an average length of 1.63 syllables $(S D=0.49)$.

In a second pretest, we determined the ease of recognizing the objects using a word-picture matching task (for other studies that used this task, see Bachoud-Lévi, Dupoux, Cohen, \& Mehler, 1998; Jescheniak \& Levelt, 1994; Meyer et al., 2007; see also Stadthagen-Gonzalez, Damian, Pérez, Bowers, \& Marin, 2009). Twenty participants saw the 24 easy objects and the 24 difficult objects as well as 48 filler objects with intermediate naming latencies. Each object was preceded by a written word. For the fillers, this was the name of the object, and for the experimental items, it was an unrelated concrete noun. The participants had to decide as quickly as possible whether the word was the name of the object. The experimental items were combined with unrelated nouns, rather than their names, to rule out priming from the word to the picture name, which could have concealed differences in the ease of recognition of the easy and difficult objects. The average decision latency was $489 \mathrm{~ms}(S D=78)$ for the easy objects and $496 \mathrm{~ms}(S D=72)$ for the difficult objects. The latency difference between the two sets of objects was not significant, $t_{1}(19)=1.01$, $p=.328 ; t_{2}(78)<1$.

In addition to the 16 experimental left objects and the 48 experimental right objects, we selected eight objects with intermediate pretest naming latencies to serve as fillers on the first eight trials of each block.

The drawings were scaled to fit into $6 \mathrm{~cm} \times 6 \mathrm{~cm}$ frames (corresponding to about $5.7^{\circ}$ when viewed from the participant's position) and were shown as black line drawings on a light gray background. The distance between the objects (center to center) was $15 \mathrm{~cm}\left(14.6^{\circ}\right)$.

Design. There were two crossed independent variables: semantic blocking (homogeneous vs. heterogeneous test blocks) and right-object difficulty (easy vs. difficult right objects). The experiment consisted of eight test blocks. Each block featured four left objects. In homogeneous blocks, they were members of the same semantic category (animals, furniture, vehicles, or tools). In heterogeneous blocks, one member of each of the four categories was presented. A different set of four objects was shown in each homogeneous block and in each heterogeneous block. By the end of the experiment, each left object had been tested in one homogeneous block and one heterogeneous block.

In each block, each left object was shown five times. As in earlier semantic blocking experiments (e.g., Belke, 2008; Belke, Meyer, \& Damian, 2005; Damian et al., 2001), the presentation of the objects was cyclic, that is, each object was shown once on Trials $1-4$, then again, in a different order, on Trials 5-8, and so on. Left objects were not repeated on successive trials. The same order of trials within a block was used for all participants.

During the first eight trials (the first two presentations of each left object), the right position on the screen was taken by one of the eight filler objects. These trials were excluded from the analyses because the semantic blocking effect takes one or two presentation cycles to build up (Belke et al., 2005).

On the following 12 trials (the third to fifth presentation of each left object), each left object was paired either with two easy objects and one difficult object, or with one difficult object and two easy objects. For instance, a participant might see the left object bed with the easy right objects scissors and tent and with the difficult 
right object piano, and they might see the left object chair with the easy right object egg and the difficult right objects slide and lungs. Each block featured six easy and six difficult right objects. Across the four homogeneous blocks, each right object was tested once. The same held for the four heterogeneous blocks. For each participant, the same pairings of left and right objects were used in the homogeneous and in the heterogeneous blocks. There were two groups of participants that differed in the pairings of left and right objects. For all participants, homogeneous and heterogeneous blocks alternated, with six participants within each group beginning with a homogeneous block and six with a heterogeneous block.

On each trial, an arrow pointing to the left or right and flanked by number symbols (\#) was shown in the center of the lower half of the screen. The two arrows appeared equally often and in random order.

Apparatus. The experiment was controlled using the Nijmegen Experimental Setup (Max Plank Institute for Psycholinguistics, Nijmegen, the Netherlands). A 19-in. (48.26-cm) Samtron 95P Plus color monitor (resolution $=42.1 \mathrm{dpi}$; refresh rate $=85$ $\mathrm{Hz}$ ) was used to present the stimuli. The participants' speech was recorded using a Sony ECM-MS907 microphone. Speech onset latencies were registered using a voice key (Hasomed $\mathrm{GmbH}$, Magdeburg, Germany). Eye movements were recorded using an SMI EyeLink I head-mounted eye-tracking system (SensoMotoric Instruments, Teltow/Berlin, Germany), which estimates the position of both eyes every $4 \mathrm{~ms}$ with a spatial accuracy of about $0.1^{\circ}$. A custom-made two-button response pad was used to record the responses to the arrows.

Procedure. Participants were tested individually in a soundattenuated booth. At the beginning of the session, they were instructed to look at a booklet showing the pictures used in the experiment and their names. To ensure good name agreement, we asked them to use only the names provided in the booklet and to refer to the objects in bare nouns (e.g., "apple," "flag").

The headband of the eye tracker was then positioned on the participant's head, and the eye-tracking system was calibrated. At the beginning of each trial, a fixation cross was presented in the center of the top left quadrant of the screen for $800 \mathrm{~ms}$. Then an object pair and an arrow were displayed for $4 \mathrm{~s}$. This was followed by an intertrial interval of $1,250 \mathrm{~ms}$. The participants were instructed to fixate on the fixation cross, to name the objects (starting with the left one), and, finally, to categorize the arrow as pointing to the left or right, using the response pad. They should use the left index finger and left button to indicate that the arrow pointed to the left, and the right index finger and right button to indicate that it pointed to the right. There were short breaks between the blocks. The experimental session lasted approximately $40 \mathrm{~min}$.

Dependent measures and data analysis. The cognitive parsing algorithm of the EyeLink software was used to determine the average positions and durations of the participants' fixations. Fixations were categorized as falling on one of the objects when they occurred within a virtual frame of $6 \mathrm{~cm} \times 6 \mathrm{~cm}\left(5.7^{\circ} \times 5.7^{\circ}\right)$ around the center of the object. They were categorized as being on the arrow when they fell within a virtual frame of $1 \mathrm{~cm} \times 1 \mathrm{~cm}$ $\left(0.9^{\circ} \times 0.9^{\circ}\right)$ of the arrow.

We determined in which order the participants looked at the objects and the arrow. We included only those trials in the main analyses on which the stimuli had been inspected in the expected order (left object - right object - arrow; see the Results section for details). As in the studies by Morgan and Meyer (2005) and Meyer et al. (2008), the most important dependent variables were the first-pass gaze durations (gaze durations hereafter) for the left and right objects. These corresponded to the first fixation duration when there was only one fixation to an object and to the time between the onset of the first fixation and the offset of the last fixation when there were two or more successive fixations to an object.

In addition to the gaze durations, we determined the error rates as the proportions of trials on which participants named one of the objects incorrectly, hesitated, stuttered, or repaired the utterances, and we measured the utterance onset latencies, which corresponded to the naming latencies for the left object. As in the studies by Morgan and Meyer (2005) and by Meyer et al. (2008), speech onset latencies for the right-object names were not recorded because they depend not only on the time required to plan the name of the right object but also on the spoken duration of the name of the left object. The speech onset latency is therefore a less precise measure of the processing time for the right object than the gaze duration. Finally, we determined when the participants looked at the arrow and when they categorized it, measured from display onset. ${ }^{1}$

For each dependent measure, analyses of variance with participants and items as random variables were conducted, yielding $F_{1}$ and $F_{2}$ statistics, respectively. As explained above, semantic blocking and right-object difficulty were tested within participants. In the by-item analyses of error rates, speech onset latencies, and left-object gaze durations, the 16 left items were used as units of analysis, and semantic blocking and right-object difficulty were within-items variables. In the by-item analyses of right-object gaze durations, the 48 right objects were used as units of analysis. Here, semantic blocking was a within-items variable, and right-object difficulty was a between-items variable. No by-item analyses were carried out for the arrow categorization latencies and the onset times of the gazes to the arrow.

\section{Results}

Naming errors occurred on $3.4 \%$ of the trials (see Table 1). The error rates did not differ much across experimental conditions, and analyses of variance yielded no main effect of semantic blocking or right-object difficulty and no interaction (all $F \mathrm{~s}<1$ ). On $0.35 \%$ of the trials, the response to the arrow was incorrect. Error trials were excluded from the following analyses. In addition, trials were excluded when the speech onset latency exceeded $2 \mathrm{~s}(1.22 \%$ of trials) or when the voice key was triggered by noise in the environment ( $1.82 \%$ of trials).

As expected, the participants usually looked first at the left object, then at the right object, and finally at the arrow. On $0.08 \%$ of the trials, they failed to look at the right object or the arrow, and on $9.00 \%$ of the trials, they did not look at the three stimuli in the expected order. On these trials, they usually looked at the left object, then at the right object, and then again at the left object

\footnotetext{
${ }^{1}$ We also analyzed the arrow categorization latencies measured from the onset of the gaze to the arrow, but we obtained no significant differences between the experimental conditions in any of the experiments.
} 
Table 1

Results of Experiment 1: Error Rate, Speech Onset Latency, Left-Object and Right-Object Gaze Duration, Onset of Gaze to the Arrow, and Arrow Categorization Latency for Homogeneous and Heterogeneous Blocks and for Easy and Difficult Right Objects

\begin{tabular}{|c|c|c|c|c|}
\hline \multirow[b]{2}{*}{ Dependent measure } & \multicolumn{2}{|c|}{ Homogeneous blocks } & \multicolumn{2}{|c|}{ Heterogeneous blocks } \\
\hline & Easy right & Difficult right & Easy right & Difficult right \\
\hline Error rate $(\%)$ & $2.94(3.78)$ & $3.98(4.51)$ & $2.43(2.99)$ & $4.17(6.01)$ \\
\hline Speech onset latency (in $\mathrm{ms}$ ) & $876(159)$ & $886(171)$ & $847(143)$ & $849(147)$ \\
\hline Left-object gaze duration (in ms) & $579(101)$ & $561(101)$ & $542(95)$ & $518(91)$ \\
\hline Right-object gaze duration (in ms) & $472(136)$ & $536(138)$ & $469(164)$ & $549(189)$ \\
\hline Onset gaze to arrow (in ms) & $1,212(232)$ & $1,258(213)$ & $1,192(252)$ & $1,249(241)$ \\
\hline Arrow categorization latency (in ms) & $1,947(289)$ & $1,994(297)$ & $1,911(308)$ & $1,969(287)$ \\
\hline
\end{tabular}

Note. Values indicate means (and standard deviations).

before turning to the arrow $(3.62 \%$ of the trials), or they looked at the arrow before looking at the right object (5.22\% of the trials). Analyses of variance showed that the rates of unexpected gaze patterns did not differ across experimental conditions. Trials with unexpected gaze patterns were excluded from the analyses reported below. ${ }^{2}$

The results obtained for the remaining trials are summarized in Table 1. As expected, the participants were slower (by $33 \mathrm{~ms}$ ) to initiate the utterances in homogeneous blocks $(M=881 \mathrm{~ms})$, in which all left objects belonged to the same semantic category, than in heterogeneous blocks $(M=848 \mathrm{~ms})$, in which they belonged to different semantic categories. The mean speech onset latencies were very similar for the easy right-object condition and the difficult right-object condition (862 vs. $868 \mathrm{~ms}$ ). In other words, the speech onset latencies depended on the ease of retrieving the first object name but not on the ease of retrieving the second object name. The analyses of variance of the speech onset latencies yielded only a main effect of semantic blocking, $F_{1}(1,23)=$ $16.15, p<.001 ; F_{2}(2,15)=17.36, p<.001$. There was no main effect of right-object difficulty and no interaction of semantic blocking and right-object difficulty (all $F_{\mathrm{s}}<1$ ).

Paralleling the results obtained for the speech onset latencies, the average duration of the gazes to the left object was also longer in homogeneous than in heterogeneous blocks $(570 \mathrm{~ms}$ vs. 530 $\mathrm{ms})$. In addition, the average left-object gaze durations depended on the difficulty of the right object: They were longer, by $20 \mathrm{~ms}$, when the right object was easy $(M=560 \mathrm{~ms})$ than when it was difficult $(M=540 \mathrm{~ms})$. The analyses of variance of the gaze durations yielded significant main effects of semantic blocking, $F_{1}(1,23)=22.74, p<.001 ; F_{2}(1,15)=16.44, p<.001$, and right-object difficulty, $F_{1}(1,23)=10.26, p=.004 ; F_{2}(1,15)=$ $4.47, p=.052$. The interaction of these variables was not significant, $F_{1}(1,23)<1 ; F_{2}(1,15)=1.16, p=.301$.

As expected, the participants looked longer at difficult than at easy right objects ( $M \mathrm{~s}=471 \mathrm{~ms}$ vs. $543 \mathrm{~ms}$ ). The durations of the gazes to the right objects in homogeneous and heterogeneous blocks were very similar (504 ms vs. $509 \mathrm{~ms}$ ). The analyses of variance of the right-object gaze durations yielded a significant main effect of right-object difficulty, $F_{1}(1,23)=74.46, p<.001$; $F_{2}(1,30)=19.03, p<.001$, but no main effect of semantic blocking and no interaction of these variables (all $F \mathrm{~s}<1$ ).

As explained above, the participants first named the two objects and then looked at and categorized the arrow at the bottom of the screen. As they looked longer at difficult than at easy right objects, it is not surprising that looks to the arrow began later (by $52 \mathrm{~ms}$ ) in the difficult right-object condition than in the easy right-object condition. The arrow categorization latencies (measured from picture onset) were also longer (by $53 \mathrm{~ms}$ ) in the difficult right-object condition than in the easy right-object condition. In the analyses of variance, only the main effect of right-object difficulty was significant for the gaze onset times, $F_{1}(1,23)=24.49, p<.001$, or for the arrow categorization times, $F_{1}(1,23)=31.34, p<.001$. The main effect of semantic blocking was not significant, for the gaze onset times, $F_{1}<1$; for the arrow categorization latencies, $F_{1}(1,23)=1.90, p=.18$. The interaction of semantic blocking and right object difficulty was also not significant (both $F_{1} \mathrm{~s}<1$ ).

\section{Discussion}

As anticipated, the participants typically inspected the objects in the order of mention and initiated the utterance shortly after the shift of gaze from the left object to the right object. The average duration of the gazes to the left object and the average speech onset latency were longer in homogenous than in heterogeneous blocks. This replicates the results of earlier studies that used the semantic blocking paradigm (Belke \& Meyer, 2007; Damian \& Als, 2005; Kroll \& Stewart, 1994) and indicates that the selection of the names of the left objects was more time consuming when all left objects of a block were members of the same semantic category than when they belonged to different semantic categories.

The easy and difficult right objects had been selected to differ in the ease of name retrieval but were matched for ease of object recognition. Therefore, the difference in the duration of the gazes to easy versus difficult right objects demonstrates that the partic-

\footnotetext{
${ }^{2}$ In supplementary analyses, only those trials were excluded in which speakers made errors, in which one of the three stimuli was never fixated, or in which the left object was not the first object to be fixated. The results were similar to those reported above. Most importantly, for the left-object gaze durations, there was a main effect of semantic blocking $(M \mathrm{~s}=573 \mathrm{~ms}$ and $529 \mathrm{~ms}$ for homogeneous and heterogeneous blocks, respectively), $F_{1}(1,23)=26.98, p<.001 ; F_{2}(1,15)=15.78, p=.001$, and a main effect of right-object difficulty, $F_{1}(1,23)=11.55, p=.002 ; F_{2}(1,15)=$ $4.66, p=.047$, with the average gaze duration being shorter in the difficult right-object condition than in the easy right-object condition $(540 \mathrm{~ms}$ vs. $562 \mathrm{~ms}$ )
} 
ipants retrieved the name of the right object before initiating the shift of gaze to the arrow. This result is consistent with numerous other findings reviewed in the introduction showing that speakers inspect the objects they name until they have recognized them and retrieved their names (e.g., Griffin, 2001; Meyer et al., 1998; Roelofs, 2007, 2008a).

The most important result is that the participants looked longer at the left object when the name of the right object was easy than when it was difficult to retrieve. Apparently, they processed the right object before initiating the shift of gaze toward it, and this extrafoveal processing of the object was sufficient for its name to become activated. A plausible account of the finding that the average left-object gaze duration was longer when the right object was easy than when it was difficult to name is that the easy right-object names became more highly activated, or were more likely to be activated at all, and interfered more with the selection of the name of the left object than the more difficult right-object names. This account implies that the sole criterion for the overt shift of gaze from the left object to the right object was that the left object had been sufficiently processed. Alternatively, the timing of the shift of gaze might also depend on the anticipated difficulty of processing the right object. Specifically, the participants might anticipate that they would need more time to process difficult than easy right objects and might therefore initiate the overt shift of gaze earlier when the right object was difficult than when it was easy (for a related proposal, see Griffin, 2003). The participants might use such a strategy to make sure that they could produce the names of the two objects without a pause. However, because the easy and difficult right objects appeared in random order and differed in the ease of name retrieval (rather than in obvious visual features), this account also implies that the speakers accessed the name of the right object before initiating the shift of gaze toward it.

The results obtained by Meyer et al. (2008) suggest that speakers processed extrafoveal objects more efficiently when the foveated object was easy than when it was more difficult to identify and to name. Therefore, one might expect a stronger extrafovealon-foveal effect in the relatively easy heterogeneous condition than in the more difficult homogeneous condition of the present experiment. However, we only obtained main effects of semantic blocking and right-object difficulty but no interaction. This finding is further discussed in the introduction to Experiment 3.

Finally, we found that right-object difficulty affected the durations of the gazes to the left object but not the utterance onset latencies. This pattern arose because the left-object gaze durations depended only on the time required to plan the name of the left object, whereas the speech onset latencies also depended on the time speakers took before speech onset to plan the name of the right object. Speakers inspected the left object for, on average, $550 \mathrm{~ms}$ but initiated the utterance only after $865 \mathrm{~ms}$. In the intervening time period, they carried out the saccade from the left object to the right object, which took on average $65 \mathrm{~ms}$, and then they inspected the right object. They spent more time (28 $\mathrm{ms}$ ) before speech onset looking at difficult than at easy right objects. This cancelled out the opposing effect of rightobject difficulty on the durations of the gazes to the left objects and led to similar speech onset latencies for the easy and difficult right-object conditions.

\section{Experiment 2}

In Experiment 1, as in the experiments by Morgan and Meyer (2005) and Meyer et al. (2008), the critical objects appeared next to each other, just like the words in most English texts. Skilled readers often process words before fixating on them (e.g., Rayner, 1998). One might speculate that people transfer their reading strategies to the processing of the objects in a naming task and perhaps only process an object they are about to name prior to fixation if it appears to the right of the object they are currently inspecting. The aim of Experiment 2 was to assess this proposal. The same materials were used as in Experiment 1, but now the second object appeared below the first one on the left side of the screen, and the arrow appeared on the right side. As before, the participants first named the two objects and then indicated whether the arrow pointed to the left or to the right.

\section{Method}

Participants. Experiment 2 was carried out with 24 participants.

Materials, design, and procedure. The same materials and design were used as in Experiment 1 . The only difference was that the objects that had previously been shown in the top-right quadrant of the screen were now presented in the lower left quadrant, and the arrow was placed in the center of the right half of the screen (see Figure 1). The distance between the objects (center to center) was $15 \mathrm{~cm}\left(14.6^{\circ}\right)$.

The procedure was the same as in Experiment 1. The participants were asked to name the objects, now starting with the top object, and to categorize the arrow as pointing to the left or to the right.

\section{Results and Discussion}

On $5.73 \%$ of the trials, participants named one of the objects incorrectly, hesitated, or repaired their utterance (see Table 2). The error rate was higher in homogeneous than in heterogeneous blocks $(6.87 \%$ vs. $4.59 \%)$, but this difference was only significant in the analysis of item means, $F_{1}(1,23)=2.68, p=.12 ; F_{2}(1$, $15)=4.81, p=.044$. Participants made more errors when the bottom object was difficult than when it was easy $(6.41 \%$ vs. $5.05 \%)$, but this difference was also only significant in the analysis of item means, $F_{1}(1,23)=2.57, p=.112 ; F_{2}(1,15)=$ $5.22, p=.037$. The interaction of the two variables was not significant (both $F \mathrm{~s}<1$ ). Error trials were excluded from the following analyses, as were trials in which the utterance onset latency exceeded $2 \mathrm{~s}(0.35 \%$ of the trials $)$ or in which participants had made an incorrect response to the arrow $(0.22 \%$ of trials). In addition, $0.35 \%$ of the trials were excluded because the voice key was triggered by noise.

On most trials, the participants first looked at the top object, then at the bottom object, and finally at the arrow. However, on $0.7 \%$ of the trials, participants failed to look at the bottom object or the arrow, and on $16.92 \%$ of the trials, they did not look at the three regions of interest in the expected order. Most commonly, they looked at the arrow, which now appeared on the right side of the screen, before looking at the bottom object, perhaps because in reading and many other tasks, stimuli are to be processed from left 
Table 2

Results of Experiment 2: Error Rate, Speech Onset Latency, Top-Object and Bottom-Object

Gaze Duration, Onset of Gaze to the Arrow, and Arrow Categorization Latency for Homogeneous and Heterogeneous Blocks and for Easy and Difficult Bottom Objects

\begin{tabular}{lccccc}
\hline & \multicolumn{2}{c}{ Homogeneous blocks } & & \multicolumn{2}{c}{ Heterogeneous blocks } \\
\cline { 2 - 3 } \multicolumn{1}{c}{ Dependent measure } & Easy bottom & Difficult bottom & & Easy bottom & Difficult bottom \\
\hline Error rate (\%) & $6.10(7.82)$ & $7.63(8.51)$ & & $4.00(4.95)$ & $5.18(5.47)$ \\
Speech onset latency (in ms) & $892(110)$ & $905(118)$ & & $859(130)$ & $857(113)$ \\
Top-object gaze duration (in ms) & $601(158)$ & $588(146)$ & & $572(169)$ & $538(134)$ \\
Bottom-object gaze duration (in ms) & $458(150)$ & $581(162)$ & & $473(216)$ & $545(236)$ \\
Onset gaze to arrow (in ms) & $1,212(264)$ & $1,336(265)$ & & $1,193(335)$ & $1,243(313)$ \\
Arrow categorization latency (in ms) & $2,015(362)$ & $2,109(363)$ & & $1,994(394)$ & $2,035(391)$ \\
\hline
\end{tabular}

Note. Values indicate means (and standard deviations).

to right or (in the United Kingdom) in clockwise order. These trials were excluded from the analyses reported below. ${ }^{3}$

Table 2 shows the results for the remaining trials. As in Experiment 1 , participants were slower to begin to speak in homogenous than in heterogeneous blocks ( $M \mathrm{~s}=899 \mathrm{~ms}$ vs. $858 \mathrm{~ms}$ ), and again the speech onset latencies in the easy and difficult right-object conditions were very similar ( $876 \mathrm{~ms}$ and $881 \mathrm{~ms})$. In the analyses of variance, only the main effect of semantic blocking was significant, $F_{1}(1,23)=14.77, p<.001 ; F_{2}(1,15)=22.17, p<.001$; all other $F$ s $<1$.

The average duration of the gazes to the top object was also longer in homogeneous than in heterogeneous blocks (595 ms vs. $555 \mathrm{~ms}$ ). However, as in Experiment 1, there was also an effect of the difficulty of the following object: Speakers looked longer at the top object when the bottom object was easy $(M=586 \mathrm{~ms})$ than when it was difficult to name $(M=563 \mathrm{~ms})$. This replicates the extrafoveal-on-foveal effect of Experiment 1. In the analyses of variance, we obtained significant effects of semantic blocking, $F_{1}(1,23)=9.87, p=.005 ; F_{2}(1,15)=14.86, p=.002$, and bottom-object difficulty, $F_{1}(1,23)=4.81, p=.039 ; F_{2}(1,15)=$ $5.72, p=.03$. The interaction of these variables was not significant, $F_{1}(1,23)=2.11, p=.16 ; F_{2}(1,15)<1$.

The participants looked longer at difficult than at easy bottom objects. As Table 2 shows, this effect was stronger in homogenous than in heterogeneous blocks (123 and $72 \mathrm{~ms}$, respectively). Accordingly, the analyses of variance yielded a main effect of bottom object difficulty, $F_{1}(1,23)=94.86, p<.001 ; F_{2}(1,46)=26.78$, $p<.001$, and a significant interaction of semantic blocking and bottom object difficulty, $F_{1}(1,23)=4.05, p=.056 ; F_{2}(1,46)=$ 8.16, $p<.006$. There was no main effect of semantic blocking, $F_{1}<1 ; F_{2}(1,46)=2.88, p<.096$. Analyses of simple effects showed that the effect of bottom-object difficulty was significant both in homogeneous blocks, $F_{1}(1,23)=70.82, p<.001 ; F_{2}(1$, $46)=30.62, p<.001$, and in heterogeneous blocks, $F_{1}(1,23)=$ $16.65, p<.001 ; F_{2}(1,46)=10.07, p=.003$. It is not clear why this interaction, which had not been seen in Experiment 1, arose.

Similar patterns of results as for the bottom-object gaze durations were seen for the onset times of the gazes to the arrow and for the arrow categorization latencies. This is not surprising because the inspection and categorization of the arrow followed the inspection of the bottom object, and therefore any delays in the processing of the bottom object should carry forward to the process- ing of the arrow. The inspection of the arrow began later when the bottom object was difficult than when it was easy to process, and this effect was stronger in homogeneous blocks (124 ms) than in heterogeneous blocks $(50 \mathrm{~ms})$. In the analysis of variance, we obtained a significant main effect of bottom object difficulty, $F_{1}(1,23)=41.79$, $p<.001$, and a significant interaction of semantic blocking and bottom-object difficulty, $F_{1}(1,23)=11.04, p=.003$, but no main effect of semantic blocking, $F_{1}(1,23)=3.04, p=.095$.

The arrow categorization latencies were also longer when the bottom object was difficult than when it was easy, and this effect was stronger in homogeneous than in heterogeneous blocks $(91 \mathrm{~ms}$ vs. $41 \mathrm{~ms})$. The analysis of variance yielded a main effect of bottom-object difficulty, $F_{1}(1,23)=16.41, p<.001$, and a significant interaction of bottom-object difficulty and semantic blocking, $F_{1}(1,23)=6.25, p<.02$, but no main effect of semantic blocking, $F_{1}(1,23)=2.55, p=.124$.

In sum, the main results of the first two experiments are very similar. Both experiments showed that the gaze duration for the first object depended not only on the difficulty of selecting the name of that object but also on the difficulty of retrieving the name of the following object, which indicates that the name of the second object was retrieved before the saccade toward it was initiated. This was true regardless of whether the second object was shown to the right or below the first object. These results rule out that speakers only process objects prior to fixation when they are arranged in the same way as words in a typical text of their language.

\section{Experiment 3}

Meyer et al. (2008) found that extrafoveal objects were processed more efficiently when the foveated objects were easy than

\footnotetext{
${ }^{3}$ When only those trials were excluded in which participants made errors, never fixated on one of the three stimuli, or did not fixate on the top object first, the pattern of results was similar to the pattern described above. For the duration of the gazes to the top object, there was a significant effect of semantic blocking $(M \mathrm{~s}=593 \mathrm{~ms}$ and $548 \mathrm{~ms}$ for homogeneous and the heterogeneous blocks, respectively), $F_{1}(1,23)=13.98, p=.001 ; F_{2}(1$, $15)=23.41, p<.001$. The main effect of bottom-object difficulty approached significance $(M \mathrm{~s}=580 \mathrm{~ms}$ vs. $561 \mathrm{~ms}$ for easy and difficult bottom objects, respectively), $F_{1}(1,23)=3.57, p=.072 ; F_{2}(1,15)=$ 7.01, $p=.018$.
} 
when they were more difficult to process. Therefore, one might expect that in Experiments 1 and 2 of the present study, the difficulty of the second object should have a stronger effect in the relatively easy heterogeneous condition than in the more difficult homogeneous condition. The condition means showed the expected pattern, but the interaction was not significant in either of the experiments or when both experiments were analyzed together, $F_{1}(1,46)=1.99, p=.16 ; F_{2}(1,15)=1.01, p=.333$. Contrary to the conclusion drawn by Meyer et al., this might suggest that the speakers' allocation of visual attention to the two objects is independent of the difficulty of the foveated object. Alternatively, the manipulation of first-object difficulty in Experiments 1 and 2 may have been too weak to affect the processing of the second object, or it may have been of the wrong type: Perhaps the distribution of the speakers' visual attention is affected by visual-conceptual variables, which were varied in the study by Meyer et al., but not by the ease of lexical access, which was varied in the first two experiments of the present study.

The goal of Experiment 3 was to determine whether the size of the extrafoveal-on-foveal effect would be reduced when the visual-conceptual processing of the foveated objects was rendered more difficult. The same spatial arrangement of the objects was used as in Experiment 1, but the recognition of the left objects was rendered more difficult by presenting them upside down (see Figure 1). If under these conditions the extrafoveal object is processed less extensively, the extrafoveal-on-foveal effect should be attenuated or absent.

\section{Method}

Participants. The experiment was carried out with 24 participants.

Materials and design. The two objects were shown next to each other, as in Experiment 1. For the left position, 12 of the 16 experimental pictures of the preceding experiments were used. The four pictures of tools were replaced by new items (items of clothing) because they do not have an obvious canonical orientation and cannot be shown upside down. For the right position, the same objects were used as in Experiments 1 and 2, except that replacement was found for four objects, which were items of clothing (see the Appendix).

The design was the same as in Experiments 1 and 2. As before, the first two presentation cycles in each block (the first eight trials, featuring each left picture twice) were to be excluded from the analyses. On these trials, the left objects were presented in their canonical orientation; they were only presented upside down from Trial 9 onward. This was done because a pilot study had shown that the participants quickly adapted to the unusual orientation of the objects. To maximize the effect of this manipulation, it was only introduced when the experimental trials began.

Apparatus. Because of technical problems, new equipment had to be used for this experiment. The eye movements were recorded using an SR EyeLink II head-mounted eye tracker, which has the same spatial resolution (about $0.1^{\circ}$ ) as the EyeLink I eye tracker used before. The same sampling rate $(250 \mathrm{~Hz})$ was used. The experiment was controlled by the software ExperimentBuilder, provided by SR Research. The headsets of the two systems and the routine of calibrating the system are almost identical. The same color monitor as in the preceding experiments was used to present the stimuli. The audio equipment was also the same. Finally, the experiment was conducted by the same experimenter in the same experimental room as Experiments 1 and 2.

Procedure. The procedure was the same as in Experiment 1, except that the eye tracker was now recalibrated after each block.

\section{Results and Discussion}

Naming errors, disfluencies, and repairs occurred on $3.91 \%$ of the trials (see Table 3). The participants made more errors in homogeneous than in heterogeneous blocks (4.86\% vs. $3.74 \%$ ), and they made more errors when the right object was difficult $(5.39 \%)$ than when it was easy $(2.43 \%)$. In the analyses of variance, the main effect of semantic blocking was only significant in the analysis by participants, $F_{1}(1,23)=8.14, p=.009 ; F_{2}(1$, $15)=1.48, p=.243$, whereas the effect right-object difficulty was significant in both analyses, $F_{1}(1,23)=17.46, p<.001 ; F_{2}(1$, $15)=17.03, p=.001$. The interaction of the two variables was not significant (both $F$ s $<1$ ).

Error trials were excluded from the analyses. In addition, trials were excluded when the voice key was triggered by noise $(1.95 \%$ of the trials) and when the naming latency exceeded $2 \mathrm{~s}$ (1.67\% of the trials). Because of a technical fault, the responses to the arrow were not recorded for four participants. For the remaining participants, the error rate for the arrow categorization task was $0.5 \%$. These trials were excluded from the analyses. Finally, $2.43 \%$ of the trials were excluded because participants failed to look at one of

Table 3

Results of Experiment 3: Error Rate, Speech Onset Latency, Left-Object and Right-Object Gaze

Duration, Onset of Gaze to the Arrow, and Arrow Categorization Latency for Homogeneous and Heterogeneous Blocks and for Easy and Difficult Right Objects

\begin{tabular}{|c|c|c|c|c|}
\hline \multirow[b]{2}{*}{ Dependent measure } & \multicolumn{2}{|c|}{ Homogeneous blocks } & \multicolumn{2}{|c|}{ Heterogeneous blocks } \\
\hline & Easy right & Difficult right & Easy right & Difficult right \\
\hline Error rate $(\%)$ & $3.30(4.07)$ & $6.42(6.50)$ & $1.56(2.96)$ & $4.36(5.29)$ \\
\hline Speech onset latency (in ms) & $916(127)$ & 924 (124) & $890(116)$ & $897(113)$ \\
\hline Left-object gaze duration (in ms) & $730(200)$ & $727(182)$ & 696 (187) & $689(174)$ \\
\hline Right-object gaze duration (in ms) & $522(178)$ & $599(166)$ & $516(155)$ & $604(156)$ \\
\hline Onset gaze to arrow (in ms) & $1,419(310)$ & $1,489(294)$ & $1,382(293)$ & $1,461(293)$ \\
\hline Arrow categorization latency (in ms) & $2,129(431)$ & $2,217(398)$ & $2,066(374)$ & $2,178(368)$ \\
\hline
\end{tabular}

Note. Values indicate means (and standard deviations). 
the regions of interest, and $8.80 \%$ of the trials were excluded because they did not inspect the objects and the arrow in the expected order.

As in Experiments 1 and 2, the average utterance onset latencies were longer in homogeneous than in heterogeneous blocks (920 ms vs. $894 \mathrm{~ms}$ ), and they were very similar in the easy and difficult right-object conditions (903 $\mathrm{ms}$ and $910 \mathrm{~ms}$ ). The analyses of variance yielded a significant main effect of semantic blocking, $F_{1}(1,23)=5.01, p=.035 ; F_{2}(1,15)=7.59, p=.015$, but no significant effect of right-object difficulty, $F_{1}(1,23)=3.09, p=$ $.092 ; F_{2}(1,15)<1$, and no interaction (both $F_{\mathrm{s}}<1$ ).

Parallel results were obtained for the duration of the gazes to the left object. The average gaze duration was longer in homogeneous than in heterogeneous blocks ( $729 \mathrm{~ms}$ vs. $693 \mathrm{~ms}$ ), but the means were similar for the easy and difficult right-object conditions (713 ms vs. $708 \mathrm{~ms}$ ). Thus, the extrafoveal-on-foveal effect seen in Experiments 1 and 2 was absent in the present experiment. In the analysis of variance, we only obtained a significant main effect of semantic blocking, $F_{1}(1,23)=8.89, p=.007 ; F_{2}(1,15)=5.84$, $p=.029$. There was no significant effect of right-object difficulty and no interaction of semantic blocking and right-object difficulty (all $F_{\mathrm{s}}<1$ ).

As expected, the participants looked longer at difficult than at easy right objects (602 ms vs. $519 \mathrm{~ms}$ ). The right-object gaze durations in homogeneous and heterogeneous blocks were almost identical (561 ms vs. $560 \mathrm{~ms}$ ). In the analysis of variance, only the main effect of right-object difficulty was significant, $F_{1}(1,23)=$ 59.07, $p<.001 ; F_{2}(1,46)=26.48, p<.001$; all other $F \mathrm{~s}<1$.

As in the preceding experiments, the effect of the difficulty of second object carried forward to the onset times of the gazes to the arrow and to the categorization latencies. The participants looked significantly later (by $86 \mathrm{~ms}$ ) at the arrow when the right object was difficult than when it was easy, $F_{1}(1,23)=56.46, p<.001$. The effect of semantic blocking and the interaction of semantic blocking and right-object difficulty were not significant, $F_{1}(1$, $23)=1.33, p=.260$, and $F_{1}<1$, respectively. The arrow categorization latencies were also significantly longer (by $80 \mathrm{~ms}$ ) when the right object was difficult than when it was easy, $F_{1}(1$, $19)=29.07, p<.001$. The main effect of semantic blocking and the interaction of the two variables were not significant: for the effect of semantic blocking, $F_{1}(1,19)=3.19, p=.090$; for the interaction, $F_{1}(1,19)<1$.

As Tables 1 and 3 show, the local effects of semantic blocking and right-object difficulty in Experiments 1 and 3 were very similar: The effects of semantic blocking on the average durations of the gazes to the left objects were $33 \mathrm{~ms}$ and $36 \mathrm{~ms}$ in Experiments 1 and 3, respectively; and the effects of right-object difficulty on the average durations of the gazes to the right objects were $72 \mathrm{~ms}$ and $81 \mathrm{~ms}$, respectively. The tables suggest that the participants of Experiment 3 were overall slower to process the objects than the participants of Experiment 1, but analyses of variance including the data from both experiments showed that only the difference in the durations of the gazes to the left objects was significant, $F_{1}(1,23)=23.83, p<.001 .^{4}$ This difference was expected given that the orientation of the left objects was different in the two experiments. The most important difference in the results of the two experiments is that the difficulty of the right objects affected the duration of the gazes to the left object in Experiment 1 but not in Experiment 3. In the joint analysis of the results of both experiments, this was reflected in a significant interaction, $F_{1}(1,46)=4.51, p=.039$. This indicates that the speakers processed the extrafoveal objects more extensively when the visual-conceptual processing of the foveated object was easy than when it was slightly more demanding.

\section{General Discussion}

The most important finding of the present study is that in the first two experiments, the difficulty of retrieving the name of the second object affected how long the speakers looked at the first object they had to name. Corroborating earlier findings (Meyer et al., 2008; Morgan \& Meyer, 2005), this shows that the speakers began to process the second object before initiating the saccade toward it.

We found that the saccade occurred later when the second object was easy than when it was more difficult to name. This suggests that during extrafoveal processing, easy second-object names became activated more strongly, or were more likely to be activated at all, than difficult second-object names and, therefore, interfered more with the retrieval of the name of the first object. This interference effect could arise during the selection of the lemma of the first object name (though one might then expect an interaction with the effect of semantic blocking, which was not obtained) or during the retrieval of the morphological or phonological form. It is also possible that the speakers' speech-monitoring processes were affected. When, in the easy right-object condition, the name of the second object became activated very early, participants may have needed additional time to establish the accuracy of the first object name, which delayed the saccade onset (see also Cook \& Meyer, 2008). Finally, as already discussed above, there may not have been any direct competition among the two object names; instead, the participants may have anticipated that they would need more time to process difficult than easy right objects and therefore started to look earlier at the difficult objects. Note that to use such a strategy, the participants had to process the right object quite extensively before initiating the shift of gaze toward it; otherwise, they would not know whether it was easy or difficult. Thus, regardless of its origin, the extrafoveal-on-foveal effect seen in the first two experiments demonstrates that the speakers processed the second object while fixating on the first one.

In Experiment 3, in which the left object was shown upside down, the extrafoveal-on-foveal effect was not replicated. This indicates that when, compared with the first two experiments, the processing of the foveated object was rendered more demanding, the next object was processed less extensively prior to fixation, and even easy second-object names were not activated early enough to affect the timing of the saccade toward them.

The difficulty of processing the first object was also varied within Experiments 1 and 2 by presenting the objects in semantically homogeneous versus heterogeneous blocks. Contrary to our expectation, this did not affect the size of the extrafoveal-on-foveal effect. Compared with the effect of presenting the objects in their canonical orientation versus upside down, which amounted to 160 $\mathrm{ms}$, the effect of semantic blocking on the left-object gaze dura-

\footnotetext{
${ }^{4}$ By-item analyses were not conducted because the experiments used overlapping but not identical item sets.
} 
tions was small, $40 \mathrm{~ms}$ in both experiments, and it may have been too weak to moderate the effect of right-object difficulty. An interesting alternative hypothesis to be tested in future research is that the size of extrafoveal-to-foveal effects might only depend on the difficulty of identifying the foveated objects, which differed in Experiment 1 versus Experiment 3, but not on the difficulty of lexical access, which was varied within Experiments 1 and 2. In other words, how well objects are processed prior to fixation might depend solely on visual-conceptual features of the fixated object but not on properties of their names.

The difficulty of the second object only affected the durations of the gazes to the first object but not the speech onset latencies. We have argued above that this pattern arose because the first-object gaze durations depended on the time speakers needed to process the first object to the level of the phonological form, whereas the speech onset latencies also depended on how much time they spent before speech onset processing the second object. The presence of an easy second object slowed down the processing of the first object and thereby delayed the shift of gaze from the first to the second object. However, after the shift of gaze, easy second objects were processed faster than difficult ones, either because they were intrinsically easier to process or because they had already been processed more extensively prior to the shift of gaze. This resulted in very similar speech onset latencies for the easy and difficult second-object conditions.

Dissociations between the results obtained for speech onset latencies and the durations of gazes to individual objects have been seen in other studies. They indicate that the two dependent measures are not always sensitive to the same influences (see also Roelofs, 2007). For instance, Levelt and Meyer (2000) reported that speakers looked much longer at objects when they had to describe them in adjective-noun phrases than in bare nouns, but the speech onset latencies for the two types of utterances were very similar. This pattern arose because the shift of gaze away from the target object occurred only after the speakers had generated the complete phonological form of the utterance referring to the object, which took longer for adjective-noun phrases than for bare nouns, whereas the utterance was initiated as soon as the first word had been fully planned, which took about the same amount of time for both utterance types.

What do the present results tell us about the speakers' allocation of visual attention? One account of the extrafoveal-on-foveal effects found in Experiments 1 and 2 is that the focus of the speakers' visual attention was initially directed at the first object but that the second object was processed in parallel, though with lower priority (e.g., Cave \& Bichot, 1999; LaBerge \& Brown, 1989). This account is based on results of earlier studies showing that saccade programming and shifts of visual attention are closely linked. As explained in the introduction, there is strong evidence that saccades are preceded by mandatory shifts of visual attention to the new location (e.g., Deubel \& Schneider, 1996), that the shift of visual attention to a new location occurs around the time that the programming of the corresponding saccade begins, and that closely related neural circuits are involved in saccade programming and in redirecting visual attention (e.g., Awh et al., 2006; Ikkai \& Curtis, 2008). If the programming of saccades is tightly coupled with shifts of visual attention, and if the time required to program a saccade is fairly constant, the effect of second-object difficulty on the timing of the saccades implies an effect on the timing of the shift of visual attention: Both occurred later when the second object was easy than when it was difficult to process. However, for this to be possible, the second object had to be processed in parallel with the first object; otherwise, the difficulty of the second object could not affect the timing of the shift of visual attention and the saccade. Thus, the extrafoveal-on-foveal effects seen in Experiments 1 and 2 indicate that the speakers processed the second object in parallel with the first object. In Experiment 3, the visualconceptual processing of the first object was more demanding, and therefore the extrafoveal object was processed less extensively. On the basis of the present findings, we cannot decide whether the participants in the first two experiments and the participants in the third experiment used qualitatively different processing strategiesincluding both objects in their attentional window or only on the first one- or whether they used the same basic strategy but differed in how extensively they processed the extrafoveal objects.

An alternative account of our findings is that the visual processing of the objects was always sequential. Accordingly, the speaker's covert visual attention was initially only directed at the first object. In Experiments 1 and 2, this object was easy to process, and therefore the speakers' covert attention soon (perhaps as soon as the first object had been recognized) moved to the second object so that the visual processing of the second object occurred in parallel with the retrieval of the name of the first object. When the name of the second object was easy to retrieve, it sometimes became activated before the name of the first object had been fully planned. This led to competition between the object names and delayed the saccade from the first to the second object, compared with when the second object name was more difficult to retrieve. In Experiment 3, the processing of the first object was more difficult than in Experiments 1 and 2, and therefore the shift of covert visual attention to the second object occurred later. Therefore, even easy right-object names were not activated early enough to interfere with the planning of the name of the left object, and no effect of right-object difficulty on the timing of the saccade was observed.

The parallel and the serial hypothesis both imply temporal overlap in the processing of the two objects. According to the parallel hypothesis, both objects are simultaneously attended to, which leads to parallel retrieval of their names. According to the serial hypothesis, only one object is attended to at any moment in time, but the shift of covert attention from the first to the second object occurs early enough for easy second-object names to "catch up" and interfere with the planning of the name of the first object. The two hypotheses differ in the assumptions made about the coupling of visual attention and saccade programming: The parallel hypothesis presupposes that they are tightly linked and that saccades are indicative of shifts of visual attention. By contrast, the serial hypothesis presupposes that shifts of covert attention and saccades are programmed independently of each other. On this view, the extrafoveal-on-foveal effects show that the difficulty of the second object affected the timing of the saccade toward it but not necessarily the covert shift of visual attention.

The present data do not allow us to discriminate between these hypotheses because we only know when the saccades occurred but do not have any independent indicators of the timing of the shifts of visual attention. On the grounds of plausibility and parsimony, we favor the parallel hypothesis. We find it more plausible than the serial hypothesis because shifts of visual attention and saccade programming are usually tightly linked. To begin to attend to the second object early, while maintaining fixation on the first object, speakers would have to cancel or suspend the planned eye movement to the 
second object. This may be effortful (e.g., Kelley, Serences, Giesbrecht, \& Yantis, 2008; Van der Stigchel \& Theeuwes, 2007), and it is not clear what would be gained from using such a processing strategy. Why should speakers redirect their visual attention without an accompanying eye movement? We find the parallel hypothesis more parsimonious because it implies that a single criterion governs the timing of covert attention shifts and overt eye movements. Earlier research reviewed in the introduction (e.g., Roelofs, 2007, 2008a) suggests that this criterion is likely to be linked to the onset of phonological encoding of the present object's name. By contrast, when shifts of visual attention and saccade programming are decoupled, as they are under the serial hypothesis, separate criteria need to be postulated to govern the timing of each of them.

What follows from the present study for theories of utterance planning? We found that, as in the earlier eye-tracking studies reviewed in the introduction, the speakers usually looked at each of the objects in the order of mention until they were about to produce its name. Thus, speakers usually focus their visual attention in a highly sequential manner on the objects they name. As we have proposed in the introduction, directing visual attention to an object probably facilitates not only the recognition of the object but also the retrieval of the associated linguistic information, and attending to the objects in the order of mention may support the generation of the correct word order.

However, the results of Experiments 1 and 2 show that even though the speakers focused on the objects strictly in the sequence, there was substantial temporal overlap in the processing of the objects, arising either because of parallel visual processing of both objects or because of early shifts of visual attention from the first to the second object. This led to parallel activation of the object names.

Under which conditions object names are coactivated in everyday speech production remains to be determined. Our results show that coactivation of object names is more likely to occur when the object the speaker is currently focusing on and the following object are both easy to name. How easy it is for a speaker to name an object depends on many variables, including intrinsic properties of the objects, such as ease of recognition and name frequency, and accidental influences, such as priming by the conceptual and linguistic context. We predict that any variable that affects the difficulty of naming the foveated or extrafoveal object may affect the likelihood of their names to become coactivated (see also Mädebach, Jescheniak, \& Oppermann, 2009; Oppermann, Jescheniak, \& Schriefers, 2008). In the present experiments, in which small sets of objects were repeated many times, the processing of the objects was probably much easier than it is in most natural speech planning tasks. In addition, the consistent use of easy first objects may have encouraged the speakers to process the extrafoveal objects more intensely than they might do in most other contexts. Thus, in everyday speech planning parallel name activation, as observed here, may be the exception rather than the rule. Results obtained in a multiple-object naming study by Griffin (2001) support this suggestion: Griffin found local effects of the difficulty of naming the objects on the respective gaze durations but no evidence that the difficulty of the second object affected the durations of the gazes to the first object or the speech onset latencies. Most likely, this difference to our results arose because Griffin used a larger set of objects (48 objects for each critical position), which were not repeated and which were probably more difficult to process than the objects in the present study.
Several studies have used the picture-word interference paradigm to determine whether the phonological forms of words in different sentence positions were activated before speech onset. For instance, results reported by Meyer (1996) suggest that the phonological form of the second noun in utterances such as "the key and ball" was activated before speech onset, and results reported by Schnur, Costa, and Caramazza (2006) show that speakers retrieved the phonological form of the verb in utterances such as "The orange girl is walking" before they began to speak. These studies provide information about the speakers' planning span-how much of the utterances they prepared before speech onset-but they do not provide direct evidence about the time course of the retrieval of the words. The speakers could either retrieve two nouns, or a noun and a verb, simultaneously, or they could retrieve them in sequence and only initiate the utterance when both forms were available.

Several picture-word interference studies have investigated the time course of word form retrieval in determiner-adjective-noun phrases, such as "the green book." These studies have shown that the phonological forms of the adjective and noun are often both activated before speech onset (Costa \& Caramazza, 2002; Jescheniak \& Schriefers, 2001; Jescheniak, Schriefers, \& Hantsch, 2003; but see Schriefers \& Teruel, 1999) Again, this does not necessarily mean that they were retrieved in parallel. However, we would expect parallel activation of word forms to be more likely for an adjective-noun phrase referring to a single object than for a noun-noun conjunction referring to spatially separated objects. This is because the information to be expressed in the noun and in the adjective is presented in the same location and is therefore likely to be attended in parallel (e.g., DeSchepper \& Treisman, 1996; Roelofs, 2008b; Wühr \& Frings, 2008). Supporting this suggestion, Damian and Dumay $(2007,2009)$ demonstrated that speakers were faster to initiate adjective-noun phrases including phonologically similar words ("green gun") than dissimilar words ("blue gun"; see also Janssen et al., 2008). As Damian and Dumay (2009) argued, this effect probably arose during the phonological form of the utterances (rather than during articulatory planning) and implies that the phonological forms of the adjective and noun were simultaneously activated.

In short, more research is needed to determine under which conditions words are retrieved in parallel. The main points of the present article were to demonstrate that under favorable circumstances, the names of two objects can become activated at the same time, and more importantly, to discuss how the time course of name activation might be related to the speakers' allocation of visual attention to the objects.

\section{References}

Awh, E., Armstrong, K. M., \& Moore, T. (2006). Visual and oculomotor selection: Links, causes and implications for spatial attention. TRENDS in Cognitive Sciences, 10, 124-130.

Bachoud-Lévi, A.-C., Dupoux, E., Cohen, L., \& Mehler, J. (1998). Where is the length effect: A cross-linguistic study of speech production. Journal of Memory and Language, 39, 331-346.

Belke, E. (2008). Effects of working memory load on lexical-semantic encoding in language production. Psychonomic Bulletin \& Review, 15 357-363.

Belke, E., \& Meyer, A. S. (2007). Single and multiple object naming in healthy aging. Language and Cognitive Processes, 22, 1178-1210. 
Belke, E., Meyer, A. S., \& Damian, M. F. (2005). Refractory effects in picture naming as assessed in a semantic blocking paradigm. Quarterly Journal of Experimental Psychology: Human Experimental Psychology, 58(A), 667-692.

Brown-Schmidt, S., \& Konopka, A. E. (2008). Little houses and casas pequeñas: Message formulation and syntactic form in unscripted speech with speakers of English and Spanish. Cognition, 109, 274-280.

Brown-Schmidt, S., \& Tanenhaus, M. K. (2006). Watching the eyes when talking about size: An investigation of message formulation and utterance planning. Journal of Memory and Language, 54, 592-609.

Caramazza, A. (1997). How many levels of processing are there in lexical access? Cognitive Neuropsychology, 14, 177-208.

Cave, K. R., \& Bichot, N. P. (1999). Visuospatial attention: Beyond a spotlight model. Psychonomic Bulletin \& Review, 6, 204-223.

Cook, A. E., \& Meyer, A. S. (2008). Capacity demands of word production: New evidence from dual-task experiments. Journal of Experimental Psychology: Learning, Memory, and Cognition, 34, 886-899.

Costa, A., \& Caramazza, A. (2002). The production of noun phrases in English and Spanish: Implications for the scope of phonological encoding in speech production. Journal of Memory and Language, 46, 178198

Damian, M. F., \& Als, L. C. (2005). Long-lasting semantic context effects in the spoken production of object names. Journal of Experimental Psychology: Learning, Memory, and Cognition, 31, 1372-1384.

Damian, M. F., \& Dumay, N. (2007). Time pressure and phonological advance planning in spoken production. Journal of Memory and Language, 57, 195-209.

Damian, M. F., \& Dumay, N. (2009). Exploring phonological encoding through repeated segments. Language and Cognitive Processes, 24, 685-712.

Damian, M. F., Vigliocco, G., \& Levelt, W. J. M. (2001). Effects of semantic context in the naming of pictures and words. Cognition, 81, B77-B86.

Dell, G. S. (1986). A spreading-activation theory of retrieval in sentence production. Psychological Review, 93, 283-321.

Dell, G. S., Burger, L. K., \& Svec, W. R. (1997). Language production and serial order: A functional analysis and a model. Psychological Review, 104, 123-147.

Dell, G. S., Oppenheimer, G. M., \& Kittredge, A. K. (2008). Saying the right word at the right time: Syntagmatic and paradigmatic interference in sentence production. Language and Cognitive Processes, 23, 583608

DeSchepper, B., \& Treisman, A. (1996). Visual memory for novel shapes: Implicit coding without attention. Journal of Experimental Psychology: Learning, Memory, and Cognition, 22, 27-47.

Deubel, H., \& Schneider, W. X. (1996). Saccade target selection and object recognition: Evidence for a common attentional mechanism. Vision Research, 36, 1827-1837.

Eimer, M., van Velzen, J., Gherri, E., \& Press, C. (2007). ERP correlates of shared control mechanisms involved in saccade preparation and in covert attention. Brain Research, 1135, 145-166.

Engbert, R., Nuthmann, A., Richter, E. M., \& Kliegl, R. (2005). SWIFT: A dynamic model of saccade generation during reading. Psychological Review, 112, 779-813.

Francis, W. S., Corral, N. I., Jones, M. L., \& Sáenz, S. P. (2008). Decomposition of repetition priming components in picture naming. Journal of Experimental Psychology: General, 136, 566-590.

Gleitman, L. R., January, D., Nappa, R., \& Trueswell, J. C. (2007). On the give and take between event apprehension and utterance formulation. Journal of Memory and Language, 57, 544-569.

Griffin, Z. M. (2001). Gaze durations during speech reflect word selection and phonological encoding. Cognition, 82, B1-B14.

Griffin, Z. M. (2003). A reversed length effect in coordinating the prepa- ration and articulation of words in speaking. Psychonomic Bulletin \& Review, 10, 603-609.

Griffin, Z. M. (2004). Why look? Reasons for eye movements related to language production. In J. M. Henderson \& F. Ferreira (Eds.), The interface of language, vision, and action: What we can learn from free-viewing eye tracking (pp. 213-247). New York, NY: Psychology Press.

Griffin, Z. M., \& Bock, K. (2000). What the eyes say about speaking. Psychological Science, 11, 274-279.

Griffin, Z. M., \& Oppenheimer, D. M. (2006). Speakers gaze at objects while preparing intentionally inaccurate names for them. Journal of Experimental Psychology: Learning, Memory, and Cognition, 32, $943-$ 948.

Henderson, J. M., \& Ferreira, F. (1990). Effects of foveal processing difficulty on the perceptual span in reading: Implications for attention and eye movement control. Journal of Experimental Psychology: Learning, Memory, and Cognition, 16, 417-429.

Hoffman, J. E., \& Subramaniam, B. (1995). The role of visual attention in saccadic eye movements. Perception \& Psychophysics, 57, 787-795.

Howard, D., Nickels, L., Coltheart, M., \& Cole-Virtue, J. (2006). Cumulative semantic inhibition in picture naming: Experimental and computational studies. Cognition, 100, 464-482.

Humphreys, G. W., \& Forde, E. M. E. (2001). Hierarchies, similarity and interactivity in object recognition: "Category-specific" neuropsychological deficits. Behavioral and Brain Sciences, 24, 453-509.

Humphreys, G. W., Riddoch, M. J., \& Price, C. J. (1997). Top-down processes in object identification: Evidence from experimental psychology, neuropsychology, and functional anatomy. Philosophical Transactions: Biological Sciences, 352, 1275-1282.

Ikkai, A., \& Curtis, C. E. (2008). Cortical activity time locked to the shift and maintenance of spatial attention. Cerebral Cortex, 18, 1384-1394.

Inhoff, A. W., Starr, M., \& Shindler, K. L. (2000). Is the processing of words during eye fixations in reading strictly serial? Perception \& Psychophysics, 62, 1474-1484.

Irwin, D. E. (2004). Fixation location and fixation duration as indices of cognitive processes. In J. M. Henderson \& F. Ferreira (Eds.), The interface of language, vision, and action: What we can learn from free-viewing eye tracking (pp. 105-133). New York, NY: Psychology Press.

Irwin, D. E., \& Gordon, R. D. (1998). Eye movements, attention and trans-saccadic memory. Visual Cognition, 5, 127-155.

Janssen, N., Alario, F.-X., \& Caramazza, A. (2008). A word-order constraint on phonological activation. Psychological Science, 19, 216-220.

Jescheniak, J. D., \& Levelt, W. J. M. (1994). Word frequency effects in speech production: Retrieval of syntactic information and of phonological form. Journal of Experimental Psychology: Learning, Memory, and Cognition, 20, 824-843.

Jescheniak, J. D., Oppermann, F., Hantsch, A., Wagner, V., Mädebach, A., \& Schriefers, H. (2009). Do perceived context pictures automatically activate their phonological code? Experimental Psychology, 56, 56-65.

Jescheniak, J. D., \& Schriefers, H. S. (2001). Priming effects from phonologically related distractors in picture-word interference. Quarterly Journal of Experimental Psychology: Human Experimental Psychology, 34(A), 371-382.

Jescheniak, J. D., Schriefers, H., \& Hantsch, A. (2003). Utterance format affects phonological priming in the picture-word task: Implications for models of phonological encoding in speech production. Journal of Experimental Psychology: Human Perception and Performance, 29, 441-454.

Johnson, C. J., Paivio, A., \& Clark, J. M. (1996). Cognitive components of picture naming. Psychological Review, 120, 113-139.

Kelley, T. A., Serences, J. T., Giesbrecht, B., \& Yantis, S. (2008). Cortical mechanisms for shifting and holding visuospatial attention. Cerebral Cortex, 18, 114-125. 
Kennedy, A. (2000). Parafoveal processing in word recognition. Quarterly Journal of Experimental Psychology: Human Experimental Psychology, 53(A), 429-455.

Korvorst, M., Roelofs, A., \& Levelt, W. J. M. (2006). Incrementality in naming and reading complex numerals: Evidence from eyetracking. Quarterly Journal of Experimental Psychology, 59, 296-311.

Kroll, J. F., \& Stewart, E. (1994). Category interference in translation and picture naming: Evidence for asymmetric connections between bilingual memory representations. Journal of Memory and Language, 33, 149174.

Kuipers, J. R., \& La Heij, W. (2009). The limitations of cascading in the speech production system. Language and Cognitive Processes, 24, 120 135.

LaBerge, D. L., \& Brown, V. R. (1989). Theory of attentional operations in shape identification. Psychological Review, 96, 101-124.

Levelt, W. J. M. (1989). Speaking: From intention to articulation. Cambridge, MA: MIT Press

Levelt, W. J. M., \& Meyer, A. S. (2000). Word for word: Sequentiality in phrase generation. European Journal of Cognitive Psychology, 12, 433452

Levelt, W. J. M., Roelofs, A., \& Meyer, A. S. (1999). A theory of lexical access in language production. Behavioural and Brain Sciences, 22, $1-38$

MacKay, D. G. (1987). The organisation of perception and action: A theory for language and other cognitive skills. New York, NY: Springer.

Mädebach, A., Jescheniak, J. J., \& Oppermann, F. (2009). Ease of processing constrains the activation flow in the conceptual-lexical system during speech planning. Manuscript submitted for publication.

McDonald, S. A. (2006). Parafoveal preview benefit in reading is only obtained from the saccade goal. Vision Research, 46, 4416-4424.

Meyer, A. S. (1996). Lexical access in phrase and sentence production: Results from picture-word interference experiments. Journal of Memory and Language, 35, 477-496.

Meyer, A. S., Belke, E., Häcker, C., \& Mortensen, L. (2007). Regular and reversed word length effects in picture naming. Journal of Memory and Language, 57, 210-231.

Meyer, A. S., \& Damian, M. (2007). Activation of distracter names in the picture-picture interference paradigm. Memory \& Cognition, 35, 494503

Meyer, A. S., Ouellet, M., \& Häcker, C. (2008). Parallel processing of objects in a naming task. Journal of Experimental Psychology: Learning, Memory, and Cognition, 34, 982-987.

Meyer, A. S., Roelofs, A., \& Levelt, W. J. M. (2003). Word length effects in picture naming: The role of a response criterion. Journal of Memory and Language, 47, 131-147.

Meyer, A. S., Sleiderink, A. M., \& Levelt, W. J. M. (1998). Viewing and naming objects: Eye movements during noun phrase production. $\mathrm{Cog}$ nition, 66, B25-B33.

Meyer, A. S., \& van der Meulen, F. F. (2000). Phonological priming of picture viewing and picture naming. Psychonomic Bulletin \& Review, 7 , 314-319.

Morgan, J. L., \& Meyer, A. S. (2005). Processing of extrafoveal objects during multiple-object naming. Journal of Experimental Psychology: Learning, Memory, and Cognition, 31, 428-442.

Morsella, E., \& Miozzo, M. (2002). Evidence for a cascade model of lexical access in speech production. Journal of Experimental Psychology: Learning, Memory, and Cognition, 28, 555-563.
Navarrete, E., \& Costa, A. (2005). Phonological activation of ignored pictures: Further evidence for a cascade model of lexical access. Journal of Memory and Language, 53, 359-377.

Oppermann, F., Jescheniak, J. D., \& Schriefers, H. (2008). Conceptual coherence affects phonological activation of context objects during object naming. Journal of Experimental Psychology: Learning, Memory, and Cognition, 34, 587-601.

Papafragou, A., Hulbert, J., \& Trueswell, J. (2008). Does language guide event perception? Cognition, 108, 155-184.

Pollatsek, A., Rayner, K., \& Collins, W. E. (1984). Integrating pictorial information across eye movements. Journal of Experimental Psychology: General, 113, 426-442.

Rayner, K. (1998). Eye movements in reading and information processing: 20 years of research. Psychological Bulletin, 124, 372-422.

Reichle, E. D., Pollatsek, A., Fisher, D. I., \& Rayner, K. (1998). Towards a model of eye movement control in reading. Psychological Review, 105, $125-156$.

Reichle, E. D., Rayner, K., \& Pollatsek, A. (2003). The E-Z Reader model of eye-movement control in reading: Comparisons to other models. Behavioral and Brain Sciences, 26, 445-526.

Roelofs, A. (1992). A spreading-activation theory of lemma retrieval in speaking. Cognition, 42, 107-142.

Roelofs, A. (2007). Attention and gaze control in picture naming, word reading, and word categorization. Journal of Memory and Language, 57, 232-251.

Roelofs, A. (2008a). Attention, gaze shifting, and dual-task interference from phonological encoding in spoken word planning. Journal of Experimental Psychology: Human Perception and Performance, 34, 15801598.

Roelofs, A. (2008b). Tracing attention and the activation flow in spoken word planning using eye movements. Journal of Experimental Psychology: Learning, Memory, and Cognition, 34, 353-367.

Schnur, T. T., Costa, A., \& Caramazza, A. (2006). Planning at the phonological level during sentence production. Journal of Psycholinguistic Research, 35, 189-212.

Schriefers, H., \& Teruel, E. (1999). Phonological facilitation in the production of two-word utterances. European Journal of Cognitive Psychology, 11, 17-50.

Stadthagen-Gonzalez, H., Damian, M. F., Pérez, M. A., Bowers, J. S., \& Marin, J. (2009). Name-picture verification as a control measure for object naming: A task analysis and norms for a large set of pictures. Quarterly Journal of Experimental Psychology, 62, 1581-1597.

University of Birmingham. (2000). COBUILD Corpus of English Sentences [Database CD]. Birmingham, England: Author.

Van der Stigchel, S., \& Theeuwes, J. (2007). The relationship between covert and overt attention in endogenous cuing. Perception \& Psychophysics, 69, 719-731.

Vigliocco, G., Vinson, D. P., Damian, M. F., \& Levelt, W. J. M. (2002) Semantic distance effects on object and action naming. Cognition, 85, B61-B69.

Wühr, P., \& Frings, C. (2008). A case for inhibition: Visual attention suppresses the processing of irrelevant objects. Journal of Experimental Psychology: General, 137, 116-130.

Wühr, P., \& Waszak, F. (2003). Object-based attentional selection can modulate the Stroop effect. Memory \& Cognition, 31, 983-994. 


\section{Appendix}

\section{Materials of Experiments 1-3}

Left objects. duck, fish, mouse, snake (animals); bed, chair, desk, lamp (furniture); bike, bus, cart, train (vehicles);

Experiments 1 and 2 only: brush, drill, rake, saw (tools);

Experiment 3 only: boot, coat, hat, tie (items of clothing).

Easy right objects. basket, bone, book, button, carrot, door, ear, egg, eye, foot, hand, heart, ladder, leaf, pen, pencil, scissors, spoon, star, sun, tent;

Experiments 1 and 2 only: hat, tie, shoe;

Experiment 3 only: banana, kettle, ruler.
Difficult right objects. bucket, cactus, chain, cherries, church, crane, glasses, harp, lungs, mountain, onion, pear, piano, pin, pipe, saddle, slide, spade, toaster, toothbrush, trumpet, tweezers, yoyo; Experiments 1 and 2 only: suit;

Experiment 3 only: drill.

Filler items. apple, balloon, brush, flag, flower, key, kite, saw.

Received June 17, 2009 Revision received October 22, 2009

Accepted November 19, 2009 